\title{
Heat and spin transport in magnetic nanowires
}

\author{
Haiming Yu ${ }^{\mathrm{a}, \mathrm{b}}$, S. Granville ${ }^{\mathrm{a}}$, D.P. Yu ${ }^{\mathrm{b}}$, J.-Ph. Ansermet ${ }^{\mathrm{a}, *}$ \\ a Ecole Polytechnique Fédérale de Lausanne, IPMC, Station 3, CH-1015 Lausanne-EPFL, Switzerland

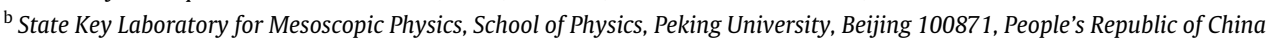

\section{A R T I C L E I N F O}

\section{Article history:}

Received 17 September 2009

Received in revised form

2 December 2009

Accepted 3 December 2009

by the Guest Editors

Available online 11 December 2009

Keywords:

A. Nanowires

B. Electrodeposition

D. Magnetogalvanic effects

D. Peltier

\begin{abstract}
A B S T R A C T
Transport measurements are carried out in which temperature oscillation is applied to magnetic nanostructures. Using spin valves, this measurement reveals aspects of the spin transport in non-collinear configurations. In one implementation, an AC voltage is detected when a DC current is driven through the nanostructure under test and its temperature is made to oscillate by illuminating it with a laser diode. A simpler approach is presented that relies on Joule heating to generate the temperature oscillation, thus eliminating the need for any optical component.
\end{abstract}

(C) 2009 Elsevier Ltd. All rights reserved.

\section{Introduction}

As a contribution to the new branch of spintronics recognized nowadays as 'spin caloritronics', we have accumulated data since 2002 concerning the interplay of heat and spin transport in magnetic nanostructures. The measurement method consists of heating up one end of a nanowire containing the magnetic nanostructure. The heating is turned on and off at frequency $f$ and the nanowire is electrically contacted. The basic idea of the measurement amounts to carrying out a thermoelectric power measurement while spins are relaxing as they cross the interface between ferromagnetic and non-magnetic metal spacers. Practically, we detect the AC voltage at the end of the nanowire, insuring that there is no AC current. Simultaneously, we drive a DC current through the nanowire. Up until now, in the measurement we called "TGV" (which stands for thermo-galvanic voltage) we shone light on one end of the membrane that contains the nanowire as a means of making its temperature oscillate. Here we report for the first time an alternative method, whereby a current at a frequency $f$ is driven, causing an oscillation of the temperature at the frequency $2 f$. The response at $2 f$ to a DC current gives similar results to TGV, but avoids the complication of the optical components required by the former method.

We report here results obtained recently with pseudo spin valves. We find that the TGV response is the same in the

\footnotetext{
* Corresponding author. Fax: +41216933603.

E-mail address: jean-philippe.ansermet@epfl.ch (J.-Ph. Ansermet).
}

parallel (P) and the anti-parallel (AP) configurations. However, as the free layer of the spin valve switches, the magnetization reaches non-collinear configurations and the TGV response as a function of applied field presents a sharp peak. Thus TGV probes parameters of transport such as spin mixing that are characteristic of non-collinear configurations. Earlier work was carried out on multilayered nanowires, where we could identify a CPP-Peltier effect [1]. The addition of a heat current to the two-current model applied to CPP transport in multilayers allows us to account for these data [2]. We showed that the spin-mixing effects could be expressed in a thermodynamic framework [3], and a generalization of heat and spin transport to non-collinear configurations was presented [4].

\section{Basic sample structure and principle of the TGV measure- ment}

Electrically contacted nanowires are prepared by the following method. Commercial membranes, containing pores of about $50 \mathrm{~nm}$ in diameter, are filled by electrodeposition from the bottom of the pores. The bottom side of the membrane (Fig. 1) is covered fully by a thick gold layer, electrically contacted to a probe holder. The other side of the membrane is only slightly covered with gold, so that the pores remain open. The potential difference between top and bottom of the pores is monitored during the growth of the nanowires.

We have the choice of stopping the growth just as the first wire makes a short between both sides of the membrane, or we can let an overgrowth form. When we want to make sure we do not have 


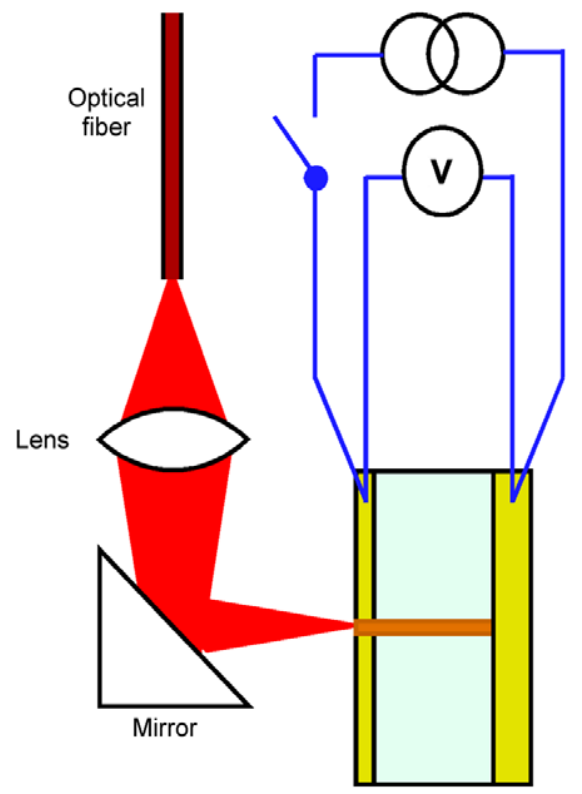

Fig. 1. Only one of many nanowires, grown in a porous membrane covered on both sides with gold, is electrically connected to a switchable current source and a voltmeter. A beam of laser light heats up the front end of the nanowire, while the end on the back side is anchored to the temperature of a massive probe holder. Typical nanowire length: $6 \mu \mathrm{m}$ [5].

a magnetically significant overgrowth, we finish the growth of the nanowire by a $\mathrm{Cu}$ deposit. The pseudo spin valves are typically $50 \mathrm{~nm}$ in diameter, the Cu spacer is about $10 \mathrm{~nm}$ thick, and the Co layers are $10 \mathrm{~nm}$ and $30 \mathrm{~nm}$ thick. This asymmetry insures distinct switching fields for the two layers, so as to obtain giant magnetoresistance (GMR).

We focus the light of a laser diode onto the membrane (Fig. 1). We can adjust the position of the laser spot until we get the maximum heating effect on the electrically connected nanowire. The heat capacity of the whole wire and its geometry are such that the nanowire thermalizes on a time scale of a few nanoseconds. Likewise, the characteristic time constant for thermalization under Joule heating is in this time scale. This was shown by numerical integration of the Fourier equation for heat, using tabulated values of the specific heat and heat conductivity [6]. The experiments below are conducted with periodic illumination at frequencies in the sub-kilohertz range, so we stand far from any transient behavior.

We have measured the isothermal resistance of uniform wires as a function of temperature. So we can determine the temperature oscillation from the observation of the resistance rise and oscillation under $\mathrm{AC}$ illumination. In order to conform to previous notation, we note $T_{A C}$ the amplitude of the temperature oscillation. We found $T_{A C}$ values of about one Kelvin, for typical power of the diode and quality of the focusing conditions of the beam onto the membrane.

The measurement method is the following: a DC current is applied to the nanowire, an oscillating illumination is applied to one face of the membrane and the voltage detected at the frequency of the light is recorded. We have called this voltage the "thermo-galvanic voltage", TGV, in our papers. After many periods of illumination, the time average of the temperature rises, but this does not affect the AC voltage. There can be also a temperature gradient which can give rise to a Seebeck contribution to the $\mathrm{AC}$ voltage. It is small because the Seebeck coefficient of $\mathrm{Cu}$, the material used as leads, is small. Furthermore, there is no magnetic field dependence of this temperature gradient. The oscillating

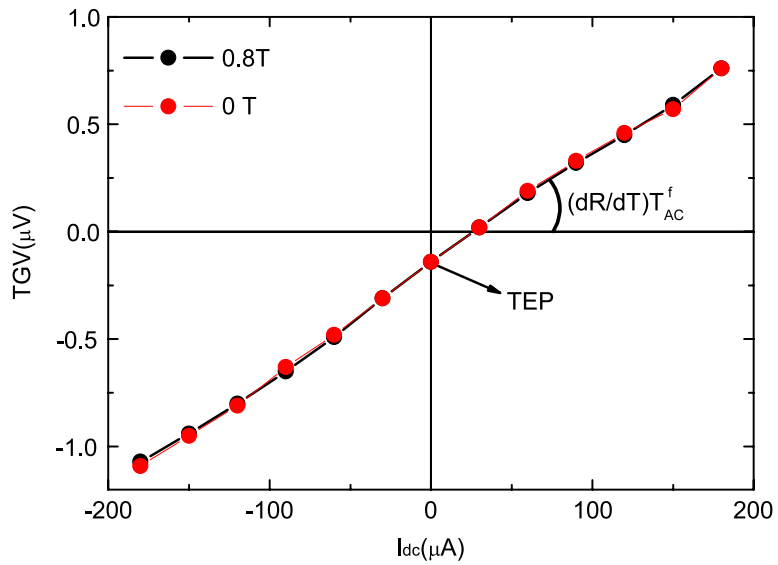

Fig. 2. AC voltage due to a temperature oscillation at the same frequency as a function of the DC current applied. This characteristic is about the same in the magnetic configurations at low and high fields. The $I_{D C}=0$ intercept is the thermoelectric power due to the small temperature gradient across the nanowire.

temperature of the nanowire can give rise to a trivial effect. Indeed, we can write:

$V_{A C}=\frac{\mathrm{d} R}{\mathrm{~d} T} T_{A C} I_{D C}$

We call isothermal resistance the resistance measured in conditions such that the temperature is uniform throughout the sample. At saturation and at zero fields, the isothermal resistance have the same temperature dependence. The data we published so far cannot be accounted for by identifying $\frac{\mathrm{d} R}{\mathrm{~d} T}$ with $\frac{\mathrm{d} R_{\text {isoth }}}{\mathrm{d} T}$, unless otherwise specified [7]. Our first results were reported for multilayered nanowires (Refs. [1,2]). In this case, we identified a contribution from the local temperature gradients that build up as a consequence of the Peltier effect when the boundary conditions are such that no heat flow is allowed at the interfaces between Co and $\mathrm{Cu}$. Since we have up to 300 interfaces, these contributions add up to a significant amount compared to the field dependence of $\frac{\mathrm{d} R_{\text {isoth }}}{\mathrm{d} T}$. That a resistance differs depending how one measures it is textbook material. Callen distinguished for example the isothermal and adiabatic resistances [8]. The existence of local temperature gradients has also been invoked by the group of Fal'ko in analyzing the thermoelectric power of metals containing ferromagnetic clusters [9].

\section{TGV of spin valves}

In the case of granular $\mathrm{Co}$ in a $\mathrm{Cu}$ film, it appeared clearly that the data could not be accounted for by Eq. (1.1) if the resistance were simply taken to be the isothermal resistance [10], despite the controversy raised [11,12]. We alluded then to the spin mixing effect as playing an important role. In order to probe with more clarity these spin mixing effects, we carried out a thermodynamic description of transport in spin valves with noncollinear magnetization vectors (Ref. [4]) and studied the TGV response of spin valves as reported below.

We characterize our spin valves by their GMR. Resistance changes as large as a fraction of an Ohm are routinely observed. Most of the resistance of the nanowire comes from the overall length of the nanowire of $\mathrm{Cu}$ leads. We measure the TGV response as a function of the DC current passing through the nanowire (Fig. 2).

We find about the same response characteristics in the $\mathrm{P}$ and the AP configurations (Fig. 2). The zero current intercept corresponds to an AC measurement of the Seebeck effect. The response is not trivial during the switching process, when one 


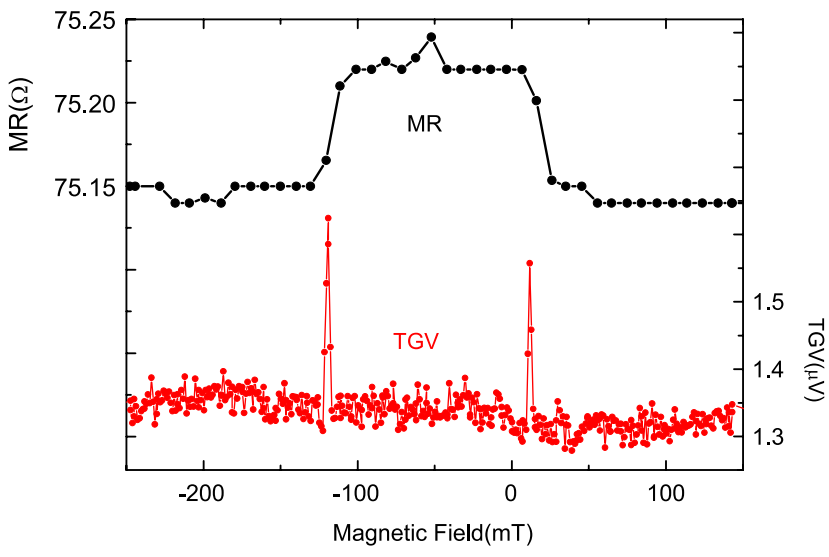

Fig. 3. Pseudo spin valve of $\mathrm{Co}-\mathrm{Cu}-\mathrm{Co}$. Top trace: $\mathrm{MR}$, bottom trace: TGV, as a function of field applied perpendicular to the nanowire (in the plane of the layers).

layer rotates with respect to the other. Then at some part of the process, we find a very sharp peak in the TGV response versus field (Fig. 3). The magnetization of two adjacent layers appears from the magnetoresistance data to undergo a reversible switching, as tested by minor loops (not shown). So, by scanning the field, we reach non-collinear configurations of the spin valve.

It is possible to account for the existence of a TGV signal in noncollinear configurations of the magnetization of spin valves using Eq. (1.1) and a model of GMR as a function of angle commonly found in the literature. There is indeed a phenomenological description of resistance as a function of the angle between the magnetization vectors of adjacent layers:

$\frac{R(\theta)-R(0)}{R(\pi)-R(0)}=\frac{1-\cos ^{2}(\theta / 2)}{1+\chi \cos ^{2}(\theta / 2)}$.

$\chi$ was first introduced as a phenomenological parameter. Then Eq. (1.2) was justified by magnetoelectronics circuit theory [13] or by using the diffusion equations for the spin accumulation in the non-colinear regime [14]. We can use our data to characterize the temperature dependence of the parameter $\chi$. Indeed, taking the temperature derivative of Eq. (1.2) yields [15]:

$\frac{1}{\chi} \frac{\mathrm{d} \chi}{\mathrm{d} T}=\frac{M T G V}{G M R}\left(\frac{1}{\rho} \frac{\mathrm{d} \rho}{\mathrm{d} T}\right)\left(\chi \frac{\mathrm{d} R_{n}\left(\theta_{\max }\right)}{\mathrm{d} \chi}\right)^{-1}$.

Here, $\rho$ is the effective resistivity of the spin valve, $\theta_{\max }$ the angle at which the magnetoresistance is maximum, GMR is the maximum change in resistance normalized to the saturation value and MTGV is the peak height of the TGV normalized by its baseline value. Using the experimental values for MTGV and GMR found for our pseudo spin valves, and owing to the fact that the last term of (1.3) is practically independent of $\chi$ if $\chi \geq 3$, we find values that lead roughly to:

$\frac{1}{\chi} \frac{\mathrm{d} \chi}{\mathrm{d} T} \approx 0.4 \frac{1}{\rho} \frac{\mathrm{d} \rho}{\mathrm{d} T}$.

For Shpiro et al. (Ref. [14]), the parameter $\chi$ is proportional to $\Lambda_{J}$, the length scale over which the transverse spin accumulation decays (Fig. 2). In this diffusive model, this term is proportional to the square root of the conductivity. This would imply: $\frac{1}{\chi} \frac{\mathrm{d} \chi}{\mathrm{d} T} \approx$ $0.5 \frac{1}{\rho} \frac{\mathrm{d} \rho}{\mathrm{d} T}$. For Brataas et al. (Ref. [13]), $\chi$ is directly proportional to the spin mixing conductance $g_{\uparrow \downarrow}$, so that $\frac{1}{\chi} \frac{\mathrm{d} \chi}{\mathrm{d} T} \approx 1.0 \frac{1}{\rho} \frac{\mathrm{d} \rho}{\mathrm{d} T}$. It would seem that our samples, as expected, are best described with a diffusive model.

At the present stage, our simplest structures constitute socalled pseudo spin valves. We refer to them as pseudo spin valves because the magnetization of one layer is not deliberately pinned. Our asymmetric deposition insures that the magnetization of each layer switches at different values of the applied field. This is easily achieved, owing to the broad distribution of switching fields in magnetic nanostructures. Further studies with exchange-biased spin valves are needed, so as to have an experimental control over the angle between the two magnetization vectors. Until these studies are conducted, we must consider alternative mechanisms, as discussed below.

There are many samples that produced TGV responses as shown in Fig. 3. The striking feature is that the TGV peak is very sharp and often stands clear of the switching as recorded by the GMR measurement. What might be happening is that the DC current sent through the spin valve under relatively high fields drives the precession of the magnetization of one of the layers. This was among the first pieces of evidence for the effect of spin polarized current on magnetization [16-18], and can also be observed in our electro-deposited samples [19]. This precession induces further dissipative processes that add a contribution to the effective resistance that could contribute to the TGV response [20]. Finally, we note that TGV measurement relies on the heating produced by the illumination obtained with a laser diode shining on one side of the membrane. Thus a temperature gradient is also applied and a heat current is driven through the nanowire. There has been prediction that the heat current can act on the magnetization [21]. It is possible that data such as those of Fig. 3 are due, in part, to this effect. In order to test this alternative, it is necessary to insure a heat current without a change in the temperature of the spin valve, so as to avoid the mechanism invoked in Eq. (1.3).

\section{Temperature oscillation by Joule heating}

Here we examine an alternative to the TGV measurement method. Instead of heating the nanowire with a laser beam, we use an AC current at a set frequency $f$, typically at less than $1 \mathrm{kHz}$. The current oscillating at frequency $f$ produces by Joule heating an oscillation of the temperature at twice the frequency. We denote by $T_{A C}^{2 f}$ the amplitude of this temperature oscillation at frequency $2 f$. By simultaneously applying a DC current, we expect to produce a voltage oscillating at $2 f$ according to:

$V_{2 f}=\frac{\mathrm{d} R}{\mathrm{~d} T} T_{A C}^{2 f} I_{D C}+\frac{\mathrm{d} R}{\mathrm{~d} M} \frac{\mathrm{d} M}{\mathrm{~d} I} I_{A C}^{2}$.

What is observed (Fig. 4) is a main contribution indeed proportional to the DC current. In addition, there is a term apparent at zero DC current, which arises from the following mechanism, reported elsewhere with more details [22]. The AC current of $100 \mu \mathrm{A}$ corresponds to a current density of about $10^{6} \mathrm{~A} / \mathrm{cm}^{2}$. It is sufficiently large to produce a torque on the magnetization, so that the resistance oscillates at the frequency of the current. Then the product of the AC current and this oscillating resistance gives rise to a voltage at twice the frequency. It gives rise also to a DC voltage which was used in an electrical detection of FMR [23]. Aside from the physical significance of the zero-current intercept, the data look very much like the characteristic function of TGV as in Fig. 2.

In particular, the data are the same at low and at high fields. However, when the measurement is carried out at a set DC current, as a function of applied magnetic field, there appear sharp peaks near the fields at which the GMR data indicate switching of one layer (Fig. 5). We have collected numerous data on similar samples. Depending on the magnetic anisotropies of a sample and the direction of the applied field, we can find such sharp peaks away from the edge of the GMR data. These instances show that these sharp peaks are not spurious effects of our detection scheme that occur because the resistance changes sharply (Ref. [22]). 


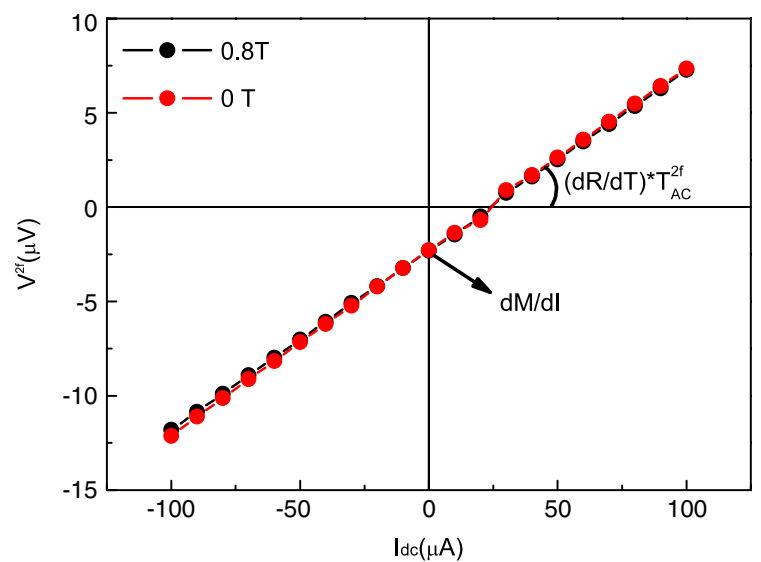

Fig. 4. Pseudo spin valve voltage detected at frequency $2 f$ as a function of the DC current $I_{D C}$ while the nanowire is subjected to a current oscillating of $100 \mu \mathrm{A}$ at frequency $f$. The zero-current intercept is due to the magnetization oscillating at frequency $f$, causing an oscillation of the resistance at the same frequency.

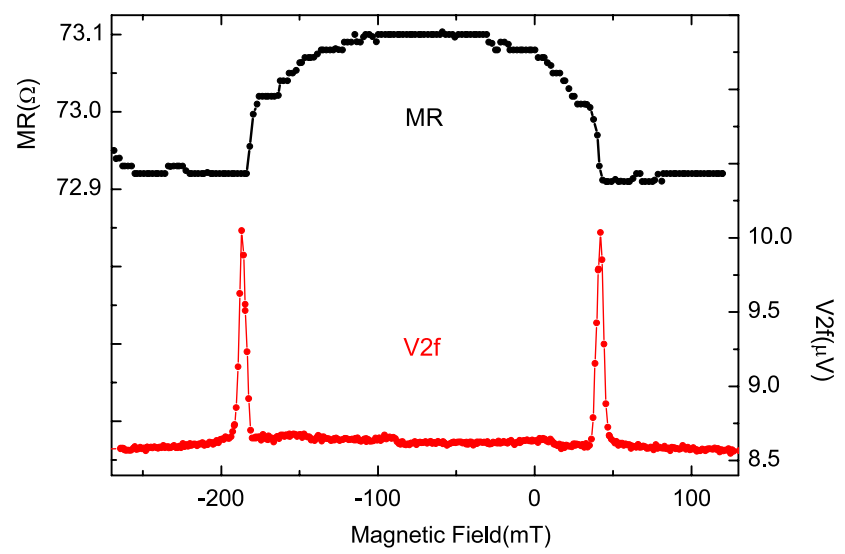

Fig. 5. Magnetoresistance (MR) and voltage response at frequency $2 f$ to an oscillating current at frequency $f$ while a DC current of $0.1 \mathrm{~mA}$ is applied to the nanowire containing a single pseudo spin valve. The field was swept from negative to positive values. This measurement is carried out on a different sample than the one used in Fig. 3.

\section{Conclusion}

TGV measurements were put in place in the wake of intensive studies of the spin transfer torque effect. The experiment introduces an oscillating temperature of the sample. Trivially, if the isothermal resistance of the wire depends on temperature then according to Eq. (1.1), this may dominate the voltage thus measured.
In pseudo spin valves, the temperature dependence of the resistance is the same in the parallel and in the antiparallel configurations. TGV, as being a measurement of $\mathrm{d} R / \mathrm{d} T$, is a sensitive test of this. However, TGV presented sharp peaks at fields near a step in the magnetoresistance, that is, when the magnetization of one layer starts rotating with respect to the other. Based on a simple parametrization of GMR as a function of the angle between the magnetization in the layers, the observed peaks are attributed tentatively to the temperature dependence of the decay length of the transverse moment.

TGV measurements have been carried out so far using a laser diode, an optical fibre and a lens to focus the light beam on the sample. Here we show that we can use an AC current to produce an oscillation of the temperature of the nanowire. The method is far simpler to implement and gives the same sharp magnetic field response. This may prove of value in sensor applications or in the readout of a magnetic memory.

\section{References}

[1] L. Gravier, S. Serrano-Guisan, F. Reuse, J.-Ph. Ansermet, Phys. Rev. B 73 (2006) 052410 .

[2] L. Gravier, S. Serrano-Guisan, F. Reuse, J.-Ph. Ansermet, Phys. Rev. B 73 (2006) 024419.

[3] J.-Ph. Ansermet, IEEE Trans. Magn. 44 (2008) 329-335.

[4] J. Dubois, J.-Ph. Ansermet, Phys. Rev. B 78 (2008) 184430.

[5] Figure L. Gravier, private communication.

[6] A. Fabian, C. Terrier, S. Serrano-Guisan, P. Guittienne, L. Gravier, J.-Ph. Ansermet, J. Phys.: Condens. Matter 18 (2006) 1569.

[7] L. Gravier, S. Serrano Guisan, G. di Domenicantionio, M. Abid, M. Hillenkamp, C. Félix, J.-Ph. Ansermet, Europhys. Lett. 77 (1) (2007) 17002.

[8] H.R. Callen, Introduction to Thermodynamics, 1st ed., John Wiley \& Sons, 1960, (Section 17.7).

[9] O. Tsyplyatyev, O. Kashuba, V.I. Fal'ko, Phys. Rev. B 74 (2006) 132403.

[10] S. Serrano-Guisan, G. di Domenicantonio, M. Abid, J.-P. Abid, M. Hillenkamp, L. Gravier, J.-Ph. Ansermet, Ch. Félix, Nature Mater. 5 (2006) 730.

[11] E.E. Fullerton, S. Mangin, Nature Materials 7 (2008) 257.

[12] S. Serrano-Guisan, G. Di Domenicantonio, M. Abid1, J.-P. Abid, M. Hillenkamp, L. Gravier1, J.Ph. Ansermet, C. Félix, Nature Mater. 7 (2008) 258.

[13] A. Brataas, G.E.W. Bauer, P.J. Kelly, Phys. Reports 427 (2006) 157.

[14] A. Shpiro, P.M. Levy, S. Zhang, Phys. Rev. B 67 (2003) 104430.

[15] S. Granville, H. Yu, J. Dubois, L. Gravier, J.-Ph. Ansermet, JMMM (in press), doi:10.1016/j.jmmm.2009.03.027.

[16] M. Tsoi, A.G.M. Jansen, J. Bass, W.-C. Chiang, M. Seck, V. Tsoi, P. Wyder, Phys. Rev. Lett. 80 (1998) 4281.

[17] E.B. Myers, D.C. Ralph, J.A. Katine, R.N. Louie, R.A. Buhrman, Science 285 (1999) 867.

[18] J.A. Katine, F.J. Albert, R.A. Buhrman, E.B. Myers, D.C. Ralph, Phys. Rev. Lett. 84 (2000) 3149.

[19] E. Murè, N. Bizière, J.-Ph. Ansermet, J. Magn. Magn. Mater. (2009) doi:10.1016/j.jmmm.2009.05.012.

[20] S. Serrano-Guisan, L. Gravier, J.-Ph. Ansermet, Mater. Sci. Eng.: B 126 (2006) 292.

[21] M. Hatami, G.E.W. Bauer, Q. Zhang, P.J. Kelly, Phys. Rev. Lett. 99 (2007) 066603.

[22] Haiming Yu, J. Dubois, S. Granville, D.P. Yu, J.-Ph. Ansermet, J. Physica D: Appl. Phys. 42 (2009) 175004.

[23] A.A. Tulapurkar, Y. Suzuki, A. Fukushima, H. Kubota, H. Maehara, K. Tsunekawa D.D. Djayaprawira, N. Watanabe, S. Yuasa, Nature 438 (2005) 339-342. 